\title{
Periodontitis as a Risk Factor for Breast Cancer - What We Know Till Date?
}

\author{
Rakhi Issrani ${ }^{1,2 *}$, RC Jagat Reddy ${ }^{2}$, Tarek H El-Metwally ${ }^{3}$, Namdeo Prabhu ${ }^{4}$
}

\begin{abstract}
A healthy microbiome is important for human health because it exhibits a variety of functions in the human body wherein the microbiome dysbiosis can lead to a variety of diseases, including cancer. Recent advances in technology and cost reduction of sequencing have made it possible and much easier for researchers to investigate the role of the microbiome in carcinogenesis. Furthermore, modulation of microflora may serve as an effective adjunct to conventional anticancer therapy that is very important to improve the patient's quality of life. Additionally, microbiome biomarkers can also be used as a diagnostic tool for cancer. So far the association between oral microbial consortia and their interactions with the host in maintaining the human health and the pathogenesis of multiple cancers has gained much popularity in the scientific research community. While the interactions of oral microflora are better established in cancer- like gastric cancer, it is far less understood in others like breast cancer. Therefore, this review briefly outlines the current information on the role of oral microbiota in breast cancer with emphasis on the mechanisms of oral microflora induced carcinogenesis and discusses the emerging role of periodontitis as a risk factor for breast cancer. Clinical relevance; Periodontitis is a very common disease that is characterized by chronic polymicrobial infection and inflammation of gingiva. It might be associated as a risk factor for breast cancer. If this association is validated in large cohort studies, it would serve as a non-invasive biomarker for breast cancer.
\end{abstract}

Keywords: Breast- cancer- microbiome- microflora- oral

Asian Pac J Cancer Prev, 22 (10), 3109-3114

\section{Introduction}

Humans are not mere individuals but a complex biological system harboring trillions of microbial cells (Parida and Sharma, 2019a; Singh et al., 2021). Human microbiome is the collective genome of all bacteria, archaea, protozoans, fungi, and viruses that inhabitants the human body which encodes nearly 100-fold more microbial genes when compared to the host genes (Parida and Sharma, 2019b; Manzoor et al., 2020; Bhatt et al., 2017). Each anatomical site of the human body exhibits a distinct and diverse microbial community that is dynamically shaped alongside human development from birth through adolescence (Manzoor et al., 2020). Human microbiome is known to colonize different sites such as the skin, the mucosa, respiratory tract, gastrointestinal tract, urogenital tract, etc (Manzoor et al., 2020). Experiments assessing gnotobiotic and germ-free mice suggest that this 'hidden and forgotten organ' provides important functions that affect cellular metabolism, inflammatory, neurological and immunologic functions (Manzoor et al.,
2020; Bhatt et al., 2017; Eslami et al., 2020; Xavier et al., 2020; Tuominen and Rautava, 2021).

Just as microbiota is important in healthy functioning, it has a hand in many disorders (Manzoor et al., 2020). Dysbiosis, defined as a disturbance in the microbiome structure, may consist of processes not necessarily mutually exclusive, like the loss of beneficial microorganisms, proliferation of pathobionts, or harmful microorganisms, and general loss of microbial diversity (Garcia-Castillo et al., 2016). Therefore, in case of disrupted regulatory circuits of host controlling homeostasis, the symbiotic relationships gets hampered, leading to a wide array of ailments ranging from neurologic to metabolic and cardiovascular disorders as well as gastrointestinal disturbances (Garcia-Castillo et al., 2016; Helmink et al., 2019).

In recent years, researchers have focused in assessing the role of microbes in carcinogenesis (Eslami et al., 2020; Sharma et al., 2021). In the year 2018, there were more than 17 million new cases of human cancer, making cancer as one of the main causes of death worldwide (Ferlay et

${ }^{1}$ Lecturer, Department of Preventive Dentistry, College of Dentistry, Jouf University, Sakaka, Kingdom of Saudi Arabia. ${ }^{2}$ Oral Medicine \& Radiology, Indira Gandhi Institute of Dental Sciences, SBV University, India. ${ }^{3}$ Professor, Medical Biochemistry Division, Department of Pathology, College of Medicine, Jouf University, Sakaka, Kingdom of Saudi Arabia. ${ }^{4}$ Department of Oral \& Maxillofacial Surgery and Diagnostic Sciences, College of Dentistry, Jouf University, Sakaka, Kingdom of Saudi Arabia. *For Correspondence: dr.rakhi.issrani00@gmail.com 
al., 2015). With an increase in population, the burden of cancer is rising when exposed to the risk factors related to cancer. Furthermore, it has been found that $15-20 \%$ of cancers are caused by infectious agents (I.IARC, 1994; Irfan et al., 2020; Chen et al., 2017; Vale, 2017). Thus, the microbiome is receiving a great deal of attention in recent years given its influence on cancer.

In regards to cancer, the microbiome falls on both the bright side and the dark side. The brighter side is improvement in the immunological system of the host by the microflora, thus decreasing the chances of cancer whereas the dark side includes their pathogenicity in causing cancer and also reducing the efficacy of anti-tumor drugs (Singh et al., 2021; Xavier et al., 2020). Furthermore, it is still debatable that whether cancer is caused by the variation in microbiota, or whether the cancerous growth leads to modifications in the normal microflora; there is strong evidence for both (Garcia-Castillo et al., 2016). On the one hand, cancerous cells exhibit different profiles compared to untransformed progenitors, and this may allow selective adhesion of other microflora. Moreover, the nutrient availability and hypoxic environment in solid tumors can favor microorganisms that require low oxygen tension. On the other hand, there is growing evidence that supports the theory that disruption of microbial communities inhabiting specific organs is directly or indirectly associated with carcinogenesis (Garcia-Castillo et al., 2016)

So far, our knowledge regarding the role of microbiome is mainly derived from gut studies as it harbors $99 \%$ of the microbial mass that helps in the gross metabolic function of the body.(Parida and Sharma, 2019b) There are fewer studies on oral microbiome and its role in carcinogenesis, especially the breast cancer. Understanding how oral microbiomes impact on breast cancer could bring new opportunities for cancer prevention, treatment, and management (Xavier et al., 2020).

Against this background, the current review was set with an aim to outline the various lines of evidence regarding the role of the oral microbiota as a risk factor in the occurrence of breast cancer, to date. In addition, we discussed the current understanding regarding pathogenesis of oral microbiota in causing cancer. Overall, these findings could give new insights for building up novel strategies for the screening or diagnosis of breast cancer and be a novel target for therapeutic approaches, thus improving the overall prognosis and quality of life of patients with breast cancer.

\section{Oral microflora}

Oral cavity contains a rich and diverse microflora like bacteria, fungi, viruses, and archaea, living in a dynamic and well-organized manner in different habitats of the oral cavity (Rautemaa et al., 2007; Yussof et al., 2020; Xu and Dongari-Bagtzoglou, 2015). The mucosa covered by keratinized and non-keratinized stratified squamous epithelium, the dorsum of the tongue and the hard structures of the teeth, consisting of supra- and sub-gingival margins are the oral habitats $(\mathrm{Xu}$ and Dongari-Bagtzoglou, 2015) Recently, the microflora of oral cavity has gained much attention in the scientific community because it is not only a influential indicator of oral health, but is also related to an increased risk of systemic diseases including gastrointestinal diseases (eg. pancreatic cancer, liver cirrhosis), neurological diseases (eg. Alzheimer's disease), endocrine system diseases (eg. obesity, diabetes, and polycystic ovary syndrome), immunological diseases (eg. rheumatoid arthritis and HIV infection), and cardiovascular diseases (eg. atherosclerosis).(Xu and Dongari-Bagtzoglou, 2015) In addition, oral microbiota has been associated with cancer development (Gao et al., 2018).

\section{Human oral microbiome database}

The findings of "Human Microbiome Project" along with the advancements in molecular techniques have revealed much information about the microflora (Chadha et al., 2021). Central to the disclosure of the oral microflora is the development of the "Human Oral Microbiome Database" (HOMD) that provides the scientific community with an integrated and collaborative information on the types of bacteria present in the oral cavity (Singh et al., 2021; Yussof et al., 2020; Teles et al., 2021). In recent years, 784 different bacterial taxa and 1,567 genomes in the human oral microbiome taxonomic hierarchy have been listed by HOMD (Tuominen and Rautava, 2021).

Few highlights regarding the oral microbiome based on the results of HOMD are as follows (Tuominen and Rautava, 2021; Yussof et al., 2020; Xu and Dongari-Bagtzoglou, 2015; Gao et al., 2018; Teles et al., 2021; Cintoni et al, 2020; Bakhti and Latifi-Navid, 2021).

- Oral microbiota has the highest $\alpha$ diversity but the lowest $\beta$ diversity when compared to other anatomical sites. Also, there are only a few varaitions in the composition of oral microbiome between different individuals;

- Bacterial diversity in the supra- and sub-gingival plaques is only second to that of the gut;

- Socransky's group first demonstrated the concept of biogeography in the oral cavity as the patterns of difference in community structure following the major anatomical habitat groups (oral, skin, gut, and vaginal);

- While studying the stability of microbial communities at different anatomical sites, the least stable site was the supra-gingival plaque, that is in consistent with the findings that the human microbiome may have been formed by an individual's recent interactions with their respective environment, as well as by medications, diet and overall health;

- The microbiome is taxonomically heterogeneous, yet functionally congruent, supporting the notion that different bacterial consortia can be compatible with a functionally homogeneous healthy ecosystem.

- Firmicutes, Bacteroidetes, Actinobacteria, Proteobacteria, and Fusobacteria forms the most prevalent phyla in the oral cavity of human adult.

\section{Changes in the oral microbiota caused by periodontitis}

Periodontitis is a disease characterized by gingival inflammation, loss of connective tissue attachment, resorption of alveolar bone, and subsequent tooth loss. (Tuominen and Rautava, 2021) In its mildest form, it is found to affect $45-50 \%$ of adults aged $>30$ years, and 
$>60 \%$ of individuals aged $>65$ years (Nguyen et al., 2000).

Irrespective of the fact that periodontitis is a multifactorial disease, microbial biofilms are a major etiological factor in chronic periodontitis (Sanz et al., 2017). Unlike most infectious diseases, periodontitis is caused by bacteria that may inhabitant the oral cavity, rather than by the formation of exogenous taxa. Thus, periodontitis might be enhanced by an overall increase in microbial biomass, rather than a change in composition of specific community. This is consistent with the findings of the increased bacterial load in mice and in people with periodontitis as they age and develop periodontitis. Additionally, qualitative changes might occur as a result of competitiveness among species that may lead to a rise in pathogenic taxa. Thus, the dynamic balance between different bacterial taxa is an important determinant in periodontal disease activity (Graves et al., 2019). Many studies have claimed an existence of a unique set of 'periodontal pathogens' that includes Aggregatibacter actinomycetemcomitans, Prevotella intermedia, P. nigrescens, Porphyromonas gingivalis, Tannerella forsythia, Treponema denticola, Parvimonas micra, Fusobacterium nucleatum, and Campylobacter rectus (Tuominen and Rautava, 2021; Lim et al., 2020).

\section{Mechanisms involved in periodontitis-linked carcinogenesis}

Several hypotheses have been put forward to explain the connecting link between periodontitis and cancer, but the basic biological mechanisms still remains unclear (Meurman, 2010). The following describes the mechanisms of carcinogenesis that are regulated commensal microorganisms (Eslami et al., 2020; Bakhti and Latifi-Navid, 2021; Meurman, 2010; Teles et al., 2020).

- Induction of chronic inflammation: Inflammatory mediators produced by oral bacteria, especially Fusobacterium, Prevotella, and Porphyromonas, cause or facilitate oncogene activation, mutagenesis, cell cycle arrest, cell proliferation, DNA damage, tumor invasiveness, migration, angiogenesis, and metastasis;

- Inhibition of the host's immune system: Oral bacteria such as $P$. gingivalis and $F$. nucleatum protect tumor cells by inhibiting immune responses. Bacterial toxins, such as A. actinomycetemcomitans-secreted cytolethal distending toxin (CDT), which exert direct DNA damage and genomic instability, can cause an imbalance of host response that affects antigen-presenting cells, and inhibit lymphocyte proliferation;

- Anti-apoptotic activity: Oral bacteria such as P. gingivalis and $\mathrm{F}$. nucleatum causes cancer growth by the activation of anti-apoptotic signaling pathways, and inhibiting pro-apoptotic pathway that affects cell proliferation, cytoskeletal rearrangement, NF- $\kappa B$ activation, and inhibits cellular apoptosis; and

- Carcinogenic substances: Oral microbiota produce certain substances that contribute to chronic inflammation, genomic instability, accumulation of mutations, metastasis, and cancer progression. For example, hydrogen sulfide and reactive oxygen species produced by Fusobacterium and Porphyromonas, respectively, are associated with colorectal neoplasia. Bacteroides and Firmicutes species ferment excessive protein of the host into sulfides and nitrosamines, which may trigger DNA alkylation and mutations. Glycosulfatase in Bacteroides can catalyze sulfomucins to release sulfides, which contributes to mucins degradation and carcinogenesis. Acetaldehyde derived from Neisseria- and Streptococcus can lead to DNA damage. Additionally, interaction of bacteria and their metabolites with toll-like receptors (TLRs) of the innate immune system are found to promote carcinogenesis in the colon, pancreas, liver, and skin.

Chronic periodontal disease can contribute to various systemic diseases via a direct and indirect route (Lim et al., 2020). Ulceration in the lining of periodontal pockets that might become a route for bacteria into the systemic circulation and eventually leading to bacteremia that facilitates the periodontal pathogens to settle in distant organs, and thus, precipitating the underlying existing diseases is the direct route (Ohki et al., 2012). The indirect route supports the theory that chronic periodontitis, that involves inflammatory pathways, may play a active role in other pathological conditions where inflammation is a major mechanism (Linden et al., 2008; Paraskevas et al., 2008). This route is strongly supported by the role of C-reactive protein in this underlying pathogenesis (Lim et al., 2020).

Some studies have reported a possible link between chronic periodontal disease and different types of cancer. (Mager et al., 2005; Nagy et al., 1998) For example, Gao et al., (2016) reported an association of $T$. denticola and $P$. gingivalis with tumorigenesis of esophageal cancer. Additionally, other studies have revealed marginal increased total cancer risks for lung, gallbladder, breast, and skin melanoma linked to chronic periodontitis (Tuominen and Rautava, 2021; Meurman, 2010).

\section{Breast cancer}

In recent years, the incidence of breast cancer has increased globally, making it the major cancer in females (Fernandez et al., 2018). In the year 2020, there were nearly 2.3 million women diagnosed with breast cancer with 685,000 deaths worldwide. By the end of 2020, there were nearly 7.8 million females alive diagnosed with breast cancer in the past 5 years, making it the most prevalent cancer globally. Also it is noteworthy that there are more lost disability-adjusted life years (DALYs) by women to breast cancer globally than any other type of cancer (WHO, 2021).

It is a multifactorial disease caused by a number of closely related risk factors; however, nearly $70 \%$ of females diagnosed with breast cancer does not have any familial risk factors (Parida and Sharma, 2019a; Parida and Sharma, 2019b). Therefore, the present risk factors and genetic-epigenetic determinants can only be confirmed by a small number of breast cancer cases globally (Chadha et al., 2021). This has led an interest among researchers in exploring the potential risk factors for breast cancer and one such factor that has recently gained much attention is the 'human microbiome' which may be an additional environmental factor related to breast cancer (Parida and Sharma, 2019a; Parida and Sharma, 2019b; Fernandez et 
al., 2018; Pellegrini et al., 2020).

The treatment for breast cancer like surgery, chemotherapy, radiotherapy, and targeted therapy may improve overall survival of a patient (Chadha et al., 2021). Unfortunately, these treatment modalities have their limitations and side-effects. In addition, these modalities target all cells non-specifically, i.e. healthy and cancerous cells that eventually leads to serious side effects like infertility, hair loss, fatigue, lowered immunity, leukemia, and depression. Overall, the side effects outweigh the benefits of cancer treatment, leading to poor prognosis and low quality of life for cancer patients (Chadha et al., 2021). Considering this, the research for exploring the novel and effective strategies that are natural and selectively cytotoxic for tumor cells are much needed. Hence, the potential health benefits of microorganisms with their ease of biomass synthesis, and the ability to synthesize a wide array of potent tumor-specific bioactive molecules render their use as a potential anti-tumor therapeutics (Chadha et al., 2021).

\section{Breast microflora}

Like any other anatomical site, the human breast also contains a different and unique bacterial community that is different from those found in other body sites (Fernandez et al, 2018). Bacteria flourish in healthy human breast tissue due to the presence of fatty tissue, extensive vasculature and lymphatic drainage that constitutes a favorable environment for their growth (Pellegrini et al., 2020). It has been proposed that these bacteria play an important role in supporting the development and maturation of the immune system in neonates (Al-Hilu et al., 2020).

Most of the available data has focused on the enormity of phyla Proteobacteria and Firmicutes in breast specimens of healthy female. Additionally, healthy women are characterized with enriched existence of Prevotella, Streptococcus, Lactococcus, Ralstonia, Corynebacterium, Sphingomonas and Micrococcus, with very low amounts of Staphylococcus, Bacteroidetes and Enterobacteriaceae. The presence of Candidatus Aquilina sp., Thermoanaerobacterium thermosaccharolyticum, Anoxybacillus, Leuconostoc, Geobacillus and Turicella otitidis have also been attributed to the healthy breast tissues.(Eslami et al., 2020; Al-Hilu et al., 2020; Urbaniak et al., 2014; Shao et al., 2018).

\section{The link between periodontitis and breast cancer}

Regarding a possible association between periodontitis and breast cancer, published studies have reported inconsistent findings, with some supporting a significant association while others claiming none. For example, a study done by Bernhard et al., (2019) to analyze the levels and proportions of 40 bacterial species in 144 sub-gingival plaque samples found an abundance of gram-negative species in the experimental group with the highest mean counts of two species viz. P. gingivalis and T. forsythia and high levels of other infectious periodontal pathogens, especially Fusobacterium and Prevotella sp. The same study concluded that an indirect pathway from periodontitis and breast cancer may be considered with periodontitis induced chronic inflammation as one of the risk factors for breast cancer. Similarly, Sfreddo et al., (2017) in a case-control study among adult Brazilian women found that patients with periodontitis had 2 to 3 times higher odds of breast cancer as compared to the women without the disease.

Freudenhaim et al., (2016) used data from 73,737 postmenopausal women who participated in the Women's Health Initiative Observational Study and after a mean follow-up of 6.7 years, reported that females with self-reported periodontitis had a $14 \%$ higher incidence of breast cancer than those without periodontitis. However, it is worth noting that self-reporting may mislead to the classification of exposure to periodontal disease that can affect the strength of this link. Chung et al., (2016) in their study reported chronic periodontitis as a risk factor for breast cancer, although no such findings were found among subjects undergoing periodontal flap surgery or gingivectomy. Soder et al., (2011) used data from 1,676 individuals and followed up for 18 years, found 2.3 higher odds of breast cancer among women with periodontitis and loss of lower molars.

Nwizu et al., (2017) used data from 65,869 postmenopausal women who participated in the Women's Health Initiative Observational Study and a maximum follow-up period of 15 years, found that a positive history of periodontal disease was associated with an increased in lung, esophagus, breast, gallbladder, and skin melanoma cancers. A prospective cohort study by Jia et al., (2020) exploring the possible association between periodontitis and invasive breast cancer and ductal carcinoma in situ (DCIS) found no clear association between periodontal disease and overall risk of breast cancer. However, an increased risk of invasive breast cancer and decreased risk of DCIS associated with periodontitis was reported. Recently, a meta-analysis was conducted with the aim of providing updated evidence on the relationship between periodontal disease and breast cancer that suggested periodontal disease as a risk factor for breast cancer. (Shao et al., 2018)

On the other hand, a study conducted by Mai et al., (2016) reported no associations between the radiographic alveolar bone loss or the presence of periodontal pathogens and total or site-specific incidence of cancer including breast cancer. Similarly, Hujoel et al., (2003) analyzed data of 11,328 individuals and followed up between 11 and 21 years during the NHANES I follow-up study, found no link between gingivitis, periodontitis or tooth loss and breast cancer. In a recent study, Wang et al., (2017) extensively examined the microbiome profiles in the breast tissue, urine, and oral rinse of breast cancer patients and compared cancerous and histologically normal tissues of cancer patients as well as cancerous and non-cancerous samples (from women undergoing cosmetic surgery). No differences were observed in terms of richness or diversity from oral rinse of all subjects.

These contradictory findings between studies can be due to the differences in study design, periodontal assessment, adjustment for confounders, follow-up time, 
and sample size (Shao et al., 2018; Sfreddo et al., 2017). Nevertheless, the relationship between oral microbiota and breast cancer remains controversial.

In conclusion, in this review, we discussed, within our scope, all available data linking periodontal disease as a risk factor for breast cancer. Till date, the findings of different studies have been inconsistent due to differences in sampling, geographical location, age, ethnicity, sampling methods, sequencing techniques, and mode of analysis. Furthermore, a link between severity of periodontal disease and breast cancer subtypes has yet to be studied. Thus, there is limited evidence of a possible link between periodontitis and breast cancer that makes it difficult to draw concrete conclusions. However, the results of few studies where periodontitis is directly or indirectly found related to breast cancer are very encouraging, but still there is much to be explored and studied.

Understanding the role of oral microflora and its modification that can reduce the incidence of breast cancer will open new strategies for the development of novel markers for early detection (or markers of susceptibility) and new methods for targeted therapeutic applications in the future. However, to emphasize their clinical translation into an effective prognostic / diagnostic marker, thorough research and complete validation of high-throughput technologies and large-scale clinical trials are much needed.

\section{Author Contribution Statement}

Conceived the ideas 4: Dr. Rakhi Issrani. Primary author (wrote most of the paper or drefted the paper): Dr. Rakhi Issrani. Provided revisions to scientific content of manuscript: Dr. Jagat reddy, Dr. Tarek H. El-Metwally Dabah. Provided grammatical revisions to manuscript: Dr. Namdeo Prabhu.

\section{Acknowledgements}

None.

\section{Conflict of interest}

None.

\section{References}

Al-Hilu SA, Al-Shujairi WH (2020). Dual role of bacteria in carcinoma: stimulation and inhibition. Int J Microbiol, 4639761. doi: 10.1155/2020/4639761.

Bakhti SZ, Latifi-Navid S (2021). Oral microbiota and Helicobacter pylori in gastric carcinogenesis: what do we know and where next?. BMC Microbiol, 21, 71.

Bernhard VR, Faveri M, Santos MS, et al (2019). Subgingival microbial profile of women with breast cancer: a cross-sectional study. Appl Cancer Res, 39, 13.

Bhatt AP, Redinbo MR, Bultman SJ (2017). The role of the microbiome in cancer development and therapy. CA Cancer J Clin, 67, 326-44.

Chadha J, Nandi D, Atri Y, Nag A (2021). Significance of human microbiome in breast cancer: tale of an invisible and an invincible. Semin Cancer Biol, 70, 112-27.
Chen J, Domingue JC, Sears CL (2017). Microbiota dysbiosis in select human cancers: evidence of association and causality. Semin Immunol, 32, 25-34.

Chung SD, Tsai MC, Huang CC, Kao LT, Chen CH (2016). A population-based study on the associations between chronic periodontitis and the risk of cancer. Int J Clin Oncol, 21, 219-23.

Cintoni M, Rinninella E, Scaldaferri F, et al (2020). The oral microbiota in oral and systemic diseases. Microb Health Dis, 2, e209.

Ferlay J, Soerjomataram I, Dikshit R, et al (2015). Cancer incidence and mortality worldwide: sources, methods and major patterns in GLOBOCAN 2012. Int J Cancer, 136, 359-86.

Eslami SZ, Majidzadeh AK, Halvaei S, Babapirali F, Esmaeili R (2020). Microbiome and breast cancer: new role for an ancient population. Front Oncol, 10, 120.

Fernandez MF, Reina-Perez I, Astorga JM, et al (2018). Breast cancer and its relationship with the microbiota. Int J Environ Res Public Health, 15, 1747.

Freudenheim JL, Genco RJ, LaMonte MJ, et al (2016). Periodontal disease and breast cancer: prospective cohort study of postmenopausal women. Cancer Epidemiol Biomarkers Prev, 25, 43-50.

Gao L, Xu T, Huang G, et al (2018). Oral microbiomes: more and more importance in oral cavity and whole body. Protein Cell, 9, 488-500.

Gao S, Li S, Ma Z, et al (2016). Presence of Porphyromonas gingivalis in esophagus and its association with the clinicopathological characteristics and survival in patients with esophageal cancer. Infect Agent Cancer, 11, 3 .

Garcia-Castillo V, Sanhueza E, McNerney E, Onate SA, Garcia A (2016). Microbiota dysbiosis: a new piece in the understanding of the carcinogenesis puzzle. $J$ Med Microbiol, 65, 1347-62.

Graves DT, Correa JD, Silva TA (2019). The oral microbiota is modified by systemic diseases. $J$ Dent Res, 98, 148-56.

Helmink BA, Khan MAW, Hermann A, Gopalakrishnan V, Wargo JA (2019). The microbiome, cancer, and cancer therapy. Nat Med, 25, 377-88.

Hujoel PP, Drangsholt M, Spiekerman C, Weiss NS (2003). An exploration of the periodontitis-cancer association. Ann Epidemiol, 13, 312-6.

I. IARC, Working group on the evaluation of carcinogenic risks to humans (1994). Biological agents. Schistosomes, liver flkes and Helicobacter pylori, IARC Monogr. Eval Carcinog Risks Hum, 61, 121-75.

Irfan M, Delgado RZR, Frias-Lopez J (2020). The oral microbiome and cancer. Front Immunol, 11, 591088.

Jia M, Wu Z, Vogtmann E, et al (2020). The association between periodontal disease and breast cancer in a prospective cohort study. Cancer Prev Res (Phila), 13, 1007-16.

Lim G, Janu U, Chiou LL, et al (2020). Periodontal health and systemic conditions. Dent J (Basel), 8, 130.

Linden GJ, McClean K, Young I, Evans A, Kee F (2008). Persistently raised C-reactive protein levels are associated with advanced periodontal disease. J Clin Periodontol, 35, 741-7.

Mager DL, Haffajee AD, Devlin PM, et al (2005). The salivary microbiota as a diagnostic indicator of oral cancer: a descriptive, non-randomized study of cancer-free and oral squamous cell carcinoma subjects. $J$ Transl Med, 3, 27.

Mai X, Genco RJ, LaMonte MJ, et al (2016). Periodontal pathogens and risk of incident cancer in postmenopausal females: the buffalo osteoperio study. $J$ Periodontol, 87, 257-67.

Manzoor SS, Doedens A, Burns MB (2020). The promise and 
challenge of cancer microbiome research. Genome Biol, 21, 131.

Meurman JH (2010). Oral microbiota and cancer. J Oral Microbiol, 2, doi: 10.3402/jom.v2i0.5195.

Nagy KN, Sonkodi I, Szoke I, Nagy E, Newman HN (1998). The microflora associated with human oral carcinomas. Oral Oncol, 34, 304-8.

Nguyen T, Brody H, Lin GH, et al (2000). Probiotics, including nisin-based probiotics, improve clinical and microbial outcomes relevant to oral and systemic diseases. Periodontol, 82, 173-85.

Nwizu NN, Marshall JR, Moysich K, et al (2017). Periodontal disease and incident cancer risk among postmenopausal women: results from the women's health initiative observational cohort. Cancer Epidemiol Biomarkers Prev, 26, 1255-65.

Ohki T, Itabashi Y, Kohno T, et al (2012). Detection of periodontal bacteria in thrombi of patients with acute myocardial infarction by polymerase chain reaction. $\mathrm{Am}$ Heart J, 163, 164-7.

Paraskevas S, Huizinga JD, Loos BG (2008). A systematic review and meta-analyses on C-reactive protein in relation to periodontitis. J Clin Periodontol, 35, 277-90.

Parida S, Sharma D (2019). The microbiome-estrogen connection and breast cancer risk. Cells, $8,1642$.

Parida S, Sharma D (2019). The power of small changes: comprehensive analyses of microbial dysbiosis in breast cancer. Biochim Biophys Acta Rev Cancer, 1871, 392-405.

Pellegrini M, Ippolito M, Monge T, et al (2020). Gut microbiota composition after diet and probiotics in overweight breast cancer survivors: a randomized open-label pilot intervention trial. Nutrition, 74, 110749.

Rautemaa R, Lauhio A, Cullinan MP, Seymour GJ (2007). Oral infections and systemic disease- an emerging problem in medicine. Clin Microbiol Infect, 13, 1041-7.

Sanz M, Beighton D, Curtis MA, et al (2017). Role of microbial biofilms in the maintenance of oral health and in the development of dental caries and periodontal diseases. Consensus report of group 1 of the Joint EFP/ORCA workshop on the boundaries between caries and periodontal disease. J Clin Periodontol, 44, S5-11.

Sfreddo CS, Maier J, De David SC, Susin C, Moreira CHC (2017). Periodontitis and breast cancer: a case-control study. Community Dent Oral Epidemiol, 45, 545-51.

Sharma VR, Singh M, Kumar V, et al (2021). Microbiome dysbiosis in cancer: exploring therapeutic strategies to counter the disease. Semin Cancer Biol, 70, 61-70.

Shao J, Wu L, Leng WD, et al (2018). Periodontal disease and breast cancer: a meta-analysis of 1,73,162 participants. Front Oncol, 8, 601.

Singh A, Nayak N, Rathi P, et al (2021). Microbiome and host crosstalk: a new paradigm to cancer therapy. Semin Cancer Biol, 70, 71-84.

Soder B, Yakob M, Meurman JH, et al (2011). Periodontal disease may associate with breast cancer. Breast Cancer Res Treat, 127, 497-502.

Teles FRF, Alawi F, Castilho RM, Wang Y (2020). Association or causation? exploring the oral microbiome and cancer links. J Dent Res, 99, 1411-24.

Teles F, Wang Y, Hajishengallis G, Hasturk H, Marchesan JT (2021). Impact of systemic factors in shaping the periodontal microbiome. Periodontol, 85, 126-60.

Thompson KJ, Ingle JN, Tang X, et al (2017). A comprehensive analysis of breast cancer microbiota and host gene expression. PLoS One, 12, e0188873.

Tuominen H, Rautava J (2021). Oral microbiota and cancer development. Pathobiology, 88, 116-26.
Urbaniak C, Cummins J, Brackstone M, et al (2014). Microbiota of human breast tissue. Appl Environ Microbiol, 80, 3007-14.

Vale S (2017). Indirect targeting of cancers via oral microbiome modifiation. J Cancer Res Immunooncol, 3, 110.

Wang H, Altemus J, Niazi F, et al (2017). Breast tissue, oral and urinary microbiomes in breast cancer. Oncotarget, $\mathbf{8 ,}$ 88122-38.

Xavier JB, Young VB, Skufca J, et al (2020). The cancer microbiome: distinguishing direct and indirect effects requires a systemic view. Trends Cancer, 6, 192-204.

$\mathrm{Xu} \mathrm{H}$, Dongari-Bagtzoglou A (2015). Shaping the oral mycobiota: interactions of opportunistic fungi with oral bacteria and the host. Curr Opin Microbiol, 26, 65-70.

Yussof A, Yoon P, Krkljes C, et al (2020). A meta-analysis of the effect of binge drinking on the oral microbiome and its relation to Alzheimer's disease. Sci Rep, 10, 19872.

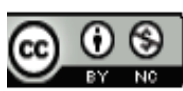

This work is licensed under a Creative Commons AttributionNon Commercial 4.0 International License. 